\title{
COMMENTARY
}

\section{Statins for acutely hospitalized patients: randomized controlled trials are long overdue}

\author{
Gordon R Bernard* \\ See related research by Christensen et al., http://ccforum.com/content/14/2/R29
}

\begin{abstract}
From the earliest studies of statins for control of plasma cholesterol, observations have been made that the reductions in mortality observed occurred in a manner seemingly independent from what could be anticipated from cholesterol lowering alone. Over the last decade, the pleiotropic effects of statins have been increasingly elucidated. Perhaps most intriguing are the effects statins appear to have on the immune system, especially the modulation of diffuse or systemic inflammation. There is a growing body of observational literature suggesting that statins can actually reduce hospital mortality through mechanisms far beyond those that can be explained by reductions in cardiovascular events.
\end{abstract}

In this issue of Critical Care, Christensen and colleagues [1] present data suggesting that patients who chronically take statins have improved survival during acute hospitalizations and for at least 1 year thereafter. Statins are now recognized to have numerous pleiotropic (Greek pleion [many] + tropos [turns]) effects. As a pharmaceutical drug class, statins inhibit the reduction of hydroxymethyl-glutaryl-CoA (HMG-CoA reductase) to mevalonate. Mevalonate is a substrate for cholesterol synthesis, hence the use of statins for control of hypercholesterolemia. Mevalonate is also involved in the synthesis of bile acids, certain steroid hormones, and vitamin D. Statins also inhibit the production of cyclooxygenase-2 (COX-2) protein and are upstream in the biosynthesis of ubiquinone (mitochondrial respiration) and heme-A (oxygen transport) and prenalation of small $\mathrm{G}$ proteins acting as molecular switches. All of these

*Correspondence: gordon.bernard@vanderbilt.edu

Department of Medicine, Vanderbilt University School of Medicine, Room T-1208, Medical Center North, Vanderbilt University, Nashville, TN 37232, USA compounds or processes have at one time or another been implicated in the altered physiology of sepsis, trauma, and other systemic injuries. It is the role of mevalonate in the prenalation of $G$ proteins that is perhaps the most far-reaching with regard to acute illness because of the profound effect this process can have on inflammatory cell activation and inflammatory protein production [2].

Beneficial effects of statins have been suggested for many diseases not directly related to cholesterol metabolism, including diabetes, multiple sclerosis, Alzheimer disease, arthritis, bone repair, anti-phospholipid syndrome, and multiple myeloma. In well-controlled animal models of pneumococcal pneumonia, statins have been shown to improve survival [3]. A recent meta-analysis of observational studies of the impact of statins in hospitalized patients by Tleyjeh and colleagues [4] showed that statins not only were associated with profoundly improved outcomes of patients admitted with infections (odds ratio 0.55, confidence interval [CI] 0.36 to 0.83 ) but also seemed to be associated with a reduction in the development of infection in high-risk patients (odds ratio 0.57 , CI 0.43 to 0.75 ).

The work by Christensen and colleagues [1] brings forward perhaps the largest and most complete observational dataset to date, and they are to be commended for providing the first data on long-term follow-up in studies of this type. These data confirm the previous work on statins demonstrating an association between the prior use of this class of drugs and reduced hospital mortality. In the present study, the population of patients is large enough to allow for robust adjustments for comorbidities, extensive subset analysis, and development of propensity scores. One of the most striking subgroups in which benefits were observed related to the particular statin (simvastatin), which is lipophilic and is the most commonly used clinically, versus the others, which were hydrophilic. The hydrophilic group was quite small, however. There is a large body of in vitro work examining the pleiotropic actions of statins according to the degree of lipophilicity versus hydrophilicity. In this regard, 
lipophilic statins seem to be more potent, but it is not clear that this will translate into better in vivo results [5].

Adding strength to the association observed by Christensen and colleagues [1], current users, new users, and long-term users derived similar benefit whereas users of other cholesterol-lowering drugs had no associated benefit and indeed effects seemed to go the wrong direction. Other subgroups that faired the best with statin use include patients with gastrointestinal disease, trauma, poisoning, cancer, or mechanical ventilation. Effects did not seem to differ as to whether the patient was surgical or medical. The survival benefit persisted out to 1 year of follow-up. At least one randomized trial in critically ill patients with infection has been completed. It was not large enough to address clinical outcomes but did demonstrate a reduction in inflammatory cytokines in the statin arm [6].

Given the results derived from observational data from a very large and still rapidly growing literature on the benefits of statins, prospective randomized trials are now clearly indicated. At least one such trial, conducted by the National Institutes of Health ARDS [Acute Respiratory Distress Syndrome] Clinical Trials Network [7], is targeting an enrollment of 1,000 patients and is under way in patients with acute lung injury (ALI) due to infection. Several aspects of statins, including the simplicity with which statins can be used in the hospital setting, their low cost, and their relative safety, make the opportunity to evaluate this class of agents in prospective trials even more exciting. These drugs have the potential to actually prevent the development of sepsis, make sepsis less severe, reduce the incidence of ALI/ARDS, and reduce mortality.

\section{Abbreviations}

ALI, acute lung injury; ARDS, acute respiratory distress syndrome; $\mathrm{Cl}$, confidence interval.

\section{Competing interests}

GRB has received consulting income and grant support from AstraZeneca (London, UK).

Published: 19 April 2010

\section{References}

1. Christensen S, Thomsen RW, Johansen MB, Pedersen L, Jensen R, Larsen KM, Larsson A, Tønnesen E, Sørensen HT: Preadmission statin use and one-year mortality among patients in intensive care: a cohort study. Crit Care 2010, 14:R29.

2. Davingnon J: Emphasis on pleiotropic effects, a new paradigm shift? Coron Artery Dis 2004, 15:223-225

3. Rosch JW, Boyd AR; Hinojosa E, Pestina T, Yunming H, Persons DA, Orihuela CJ, Tuomanen El: Statins protect against fulminant pneumococcal infection and cytolysin toxicity in a mouse model of sickle cell disease. J Clin Invest 2010, 120:627-635.

4. Tleyjeh IM, Kashour T, Hakim FA, Zimmerman VA, Erwin PJ, Sutton AJ, Ibrahim $\mathrm{T}$ : Statins for the prevention and treatment of infections. A systematic review and meta-analysis. Arch Intern Med 2009, 169:1658-1667.

5. Kiener PA, Davis PM, Murray JL, Youssef S, Rankin BM, Kowala M: Stimulation of inflammatory responses in vitro and in vivo by lipophilic HMG-CoA reductase inhibitors. Inter Immunopharm 2001, 1:105-118.

6. Novack V, Eisinger M, Frenkel A, Terblanche M, Adhikari NK, Douvdevani A, Amichay D, Almog Y: The effects of statin therapy on inflammatory cytokines in patients with bacterial infections: a randomized double-blind placebo controlled clinical trial. Int Care Med 2009, 35:1255-1260.

7. National Heart Lung and Blood Institute ARDS Network, ARDSNet Studies [http://www.ardsnet.org/studies_list].

doi:10.1186/cc8950

Cite this article as: Bernard GR: Statins for acutely hospitalized patients: randomized controlled trials are long overdue. Critical Care 2010, 14:141. 